\title{
Auditory Short-term Memory Capacity Correlates with Gray Matter Density in the Left Posterior STS in Cognitively Normal and Dyslexic Adults
}

\author{
Fiona M. Richardson ${ }^{1}$, Sue Ramsden ${ }^{1}$, Caroline Ellis ${ }^{1}$, \\ Stephanie Burnett ${ }^{1}$, Odette Megnin ${ }^{1}$, Caroline Catmur ${ }^{2}$, \\ Tom M. Schofield ${ }^{1}$, Alex P. Leff ${ }^{1}$, and Cathy J. Price ${ }^{1}$
}

\begin{abstract}
A central feature of auditory STM is its item-limited processing capacity. We investigated whether auditory STM capacity correlated with regional gray and white matter in the structural MRI images from 74 healthy adults, 40 of whom had a prior diagnosis of developmental dyslexia whereas 34 had no history of any cognitive impairment. Using whole-brain statistics, we identified a region in the left posterior STS where gray matter density was positively correlated with forward digit span, backward digit span, and performance on a "spoonerisms" task that required both auditory STM and phoneme manipulation. Across tasks and participant groups, the correlation was highly significant even when variance related to reading and auditory non-
\end{abstract}

\section{INTRODUCTION}

In cognitive theory, the most prominent property of the working memory system is its item-limited processing capacity. Early experimentation by Miller (1956) showed the maximum number of items that can be maintained in working memory in typical subjects is $7 \pm 2$ items. Although the use of encoding strategies can improve performance on memory tests with practice, experimentation suggests that the actual limitations on processing capacity in working memory cannot be increased (Ericsson, Chase, \& Faloon, 1980). In Baddeley's model, probably the most well known in the literature (Baddeley \& Hitch, 1974), working memory is characterized in terms of multiple components (such as the phonological loop and visuospatial sketchpad) that are specialized according to the type of information (i.e., verbal or visuospatial) to be temporarily stored and maintained. With the advent of neuroimaging, there have been attempts to locate the components of the working memory system within the brain to provide a mapping between cognitive theory and the neural substrates in which these processes occur (for instance, see Baddeley, 2003).

\footnotetext{
${ }^{1}$ University College London, ${ }^{2}$ University of Surrey
}

word repetition was factored out. Although the dyslexics had poorer phonological skills, the effect of auditory STM capacity in the left STS was the same as in the cognitively normal group. We also illustrate that the anatomical location of this effect is in proximity to a lesion site recently associated with reduced auditory STM capacity in patients with stroke damage. This result, therefore, indicates that gray matter density in the posterior STS predicts auditory STM capacity in the healthy and damaged brain. In conclusion, we suggest that our present findings are consistent with the view that there is an overlap between the mechanisms that support language processing and auditory STM.

Arguably, the most extensive search embarked upon has been the hunt for the phonological store-the temporary buffer in which verbal material is held. A number of functional imaging studies have suggested that the phonological store is located in the left inferior parietal cortex (see Buchsbaum \& D'Esposito, 2008, for a review). However, the actual peak coordinates reported for inferior parietal regions vary considerably — with some studies identifying a ventral inferior parietal region, whereas others report a more dorsal location (Crottaz-Herbette, Anagnoson, \& Menon, 2004; Gruber \& von Cramon, 2003; Henson, Burgess, \& Frith, 2000; Jonides et al., 1998; Awh et al., 1996; Salmon et al., 1996; Smith, Jonides, \& Koeppe, 1996; Smith et al., 1995; Paulesu, Frith, \& Frackowiak, 1993). A recent study by Ravizza, Delgado, Chein, Becker, and Fiez (2004) examining the characteristics of these two inferior parietal sites found that the ventral inferior parietal site was more sensitive to information type (verbal or nonverbal), whereas the dorsal inferior parietal region was more sensitive to information load (high vs. low). This latter finding is consistent with other studies that manipulate information load (Chen \& Desmond, 2005; Kirschen, Chen, Schraedley-Desmond, \& Desmond, 2005; Cohen et al., 1997). However, as neither site in the inferior parietal cortex displays sensitivity to both high information load and 
verbal material, the individual patterns of activation shown by these regions are not consistent with a dedicated phonological store (Ravizza et al., 2004).

In addition to parietal sites, prefrontal (Koelsch et al., 2009; Thierry, Ibarrola, Démonet, \& Cardebat, 2003; Bunge, Ochsner, Desmond, Glover, \& Gabrieli, 2001; Rypma, Prabhakaran, Desmond, Glover, \& Gabrieli, 1999) and posterior temporal regions have also been associated with verbal working memory (Hickok, Buchsbaum, Humphries, \& Muftuler, 2003). The present evidence, therefore, indicates that the components of the working memory system are distributed across multiple brain regions (D'Esposito, 2007) - a perspective that discounts the assumption of prior studies that phonological STM can be tied to a single anatomical location. Within this system, a recent study by Acheson and colleagues (2011) found that rTMS to the left posterior superior temporal gyrus (STG) resulted in an increase in error rates for language production and verbal working memory tasks. These findings in conjunction with behavioral evidence (Acheson \& MacDonald, 2009a; Page, Madge, Cumming, \& Norris, 2007) are consistent with verbal working memory being supported by the same mechanisms responsible for sequencing in language production (Acheson \& MacDonald, 2009b).

Although functional imaging can be used to identify brain regions engaged in working memory, it cannot be used to identify the neural correlates of memory capacity. This is because functional imaging measures active processing, but capacity is a constraint on processing, not a process itself. Working memory capacity is typically assessed using behavioral measures such as the digit-span task, in which participants are asked to perform immediate recall of digit sequences of increasing length (usually two to nine digits). In these tasks, capacity is indicated by the maximum number of digits that a participant can recall. Therefore, the digit-span task provides a measure of limitations in the storage and maintenance components of working memory.

Recently, two studies of patients have correlated digitspan scores with structural indices of brain damage using voxel-based morphometry (VBM). In a study of 210 stroke patients, Leff and colleagues (2009) found that the structural integrity of a region in the left posterior superior temporal gyrus predicted both auditory STM capacity (assessed using a forward digit-span task) and the ability of patients to comprehend spoken sentences. Another VBM study of 58 patients with neurodegenerative diseases carried out by Amici and colleagues (2007) used a backward digitspan task, in which digit sequences were recalled in a reverse order, placing additional processing demands on top of verbal working memory capacity and a complex sentence comprehension task (using multiclausal relative sentences). They found a positive correlation between performance on both tasks and gray matter volume in a region encompassing the left inferior and middle frontal gyri.

The structural imaging studies of Leff et al. (2009) and Amici et al. (2007) indicate that regional variance in brain structure in patient populations can be linked to perfor- mance on tasks assessing STM capacity. Although these studies identify two different regions, this difference could be explained by the use of a harder task (recall of digits in reverse order) by Amici et al. (2007). This task is more difficult, not because it places increased demands on working memory, but because it requires additional concurrent processing to reorder the digit sequence (Wilde, Strauss, \& Tulsky, 2004). Therefore, use of this task may have disproportionately increased concurrent processing demands in an impaired population of participants, which in turn engaged additional components of the working memory system in addition to the need to maintain this information within STM (Gathercole, Alloway, Willis, \& Adams, 2006). This supposition is consistent with the perspective that controlled retrieval is mediated by the inferior frontal gyrus, which is more highly activated in participants who perform more poorly on memory tasks (Buchsbaum, Padmanabhan, \& Berman, 2011).

Although the use of patient groups is advantageous when performing regression analyses, because their broad ability spectrum maximizes variance, the overall ability in patient groups is typically below the normal range. Therefore, brain regions identified in patient populations cannot be assumed to hold for participant groups that have a different range of behavioral performance. Furthermore, unlike functional imaging studies that assume equivalent power in each voxel tested, the inferences that can be drawn from lesion studies are limited to the areas where a large enough proportion of the patient group have damage (Rudrauf et al., 2008; Kimberg, Coslett, \& Schwartz, 2007).

The aim of this study was to use structural imaging and VBM to search for regions in the healthy adult brain where small differences in gray and white matter are positively correlated with auditory STM capacity. We included 40 adults with a prior diagnosis of developmental dyslexia and 34 adults with normal cognitive development (henceforth referred to as "typical" adults). Adults with a history of developmental dyslexia are known to show marginally lower performance on tests that rely on auditory STM capacity (Hatcher, Snowling, \& Griffiths, 2002; Snowling, Nation, Moxham, Gallagher, \& Frith, 1997). By including these two groups of participants, we tested whether correlations between brain structure and auditory STM capacity are consistent or different across two populations who differed in their phonological and STM abilities. In addition, we used multiple cognitive tests of auditory STM capacity, including forward and backward digit-span tasks. Tests of verbal language skills were also included to factor out variance attributable to reading skills and auditory speech perception and production across participants within each group (dyslexic or typical). This enabled us to identify brain regions that were positively correlated with auditory STM capacity while discounting variance from these sources. Finally, we used whole-brain statistics rather than ROIs to identify the most significant brain regions where variance in gray or white matter correlates with auditory STM capacity. 


\section{METHODS}

This study was approved by the joint ethical committee of the Institute of Neurology and the National Hospital for Neurology and Neurosurgery, London, United Kingdom.

\section{Participants}

Seventy-four right-handed participants took part in this study. All spoke English as their first language, were neurologically normal, and had no history of psychiatric disorder. We recruited two groups of participants, one with a diagnosis of developmental dyslexia from prior neuropsychological assessments by an educational psychologist ( $n=40 ; 23$ men), and another group without any history of cognitive impairment ( $n=34 ; 19$ men). The dyslexic group consisted mainly of participants with a subtle impairment in reading (see Table 1$)$ and significantly worse spelling than typical readers $(t(72)=$ $-6.43, p<.001)$. In accordance with previous studies (Hatcher et al., 2002; Snowling et al., 1997), the average digit-span performance of the dyslexic group was significantly lower than the typical group (see Table 1). Moreover, individuals with a history of dyslexia had a differing range of auditory STM abilities.

Further details of the participant groups, including behavioral test scores, can be seen in Table 1. A detailed description of the tests shown in this table can be found in the following section. A correlation matrix for these test scores can be found in the Appendix.

\section{Behavioral Tests}

All participants carried out a series of subtests from WAISIII (Wechsler, 1998), which included a digit-span task. Three additional behavioral assessments of verbal and phonological skills were also conducted: spoonerisms, auditory nonword repetition, and word reading. These test scores were included as covariates in our structural imaging analysis.

\section{Digit Span}

This subtest of the WAIS-III is composed of two tasks, (i) digits-forward and (ii) digits-backward, which were administered consecutively. The digits-forward task is characterized as a simple span test, measuring the storage and maintenance components of working memory. The digitsbackward task is referred to as a complex span test, as it additionally engages concurrent processing (Wilde et al., 2004). However, both tasks index verbal working memory, and scores are typically combined to provide a composite measure of performance.

The digits-forward task comprises eight difficulty levels, each containing two trials with digit sequences of the same length. Each digit sequence contains the numbers 1-9, with no digit being repeated within a sequence. The test starts with a two-digit sequence, and sequence length progressively increases with each difficulty level to a maximum length of nine digits. On each trial, the digit sequence is read aloud by the tester, and the participant then attempts

Table 1. Demographics and Behavioral Test Scores for the Two Groups of Participants

\begin{tabular}{|c|c|c|}
\hline & Typical (SD), $n=34$ & Dyslexic (SD), $n=40$ \\
\hline Age (years) & $20(4)$ & $20(4)$ \\
\hline \multicolumn{3}{|c|}{ Maximum Number of Digits Recalled ${ }^{\mathrm{a}}$} \\
\hline Forward digit-span ${ }^{\mathrm{b}}$ & $7.3(1.2)$ & $5.7(1)$ \\
\hline Backward digit-span ${ }^{\mathrm{b}}$ & $5.6(1.6)$ & $4.5(1)$ \\
\hline \multicolumn{3}{|l|}{$\%$ Correct } \\
\hline (1) Composite digit-span score ${ }^{b}$ & $66(15)$ & $49(11)$ \\
\hline (2) Spoonerisms ${ }^{b}$ & $93(10)$ & $74(29)$ \\
\hline (3) Nonword repetition & $90(8)$ & $87(11)$ \\
\hline (4) Word reading ${ }^{b}$ & $86(11)$ & $77(12)$ \\
\hline \multicolumn{3}{|l|}{$I Q$} \\
\hline Verbal IQ & $123(16)$ & $107(12)$ \\
\hline Performance IQ & $113(9)$ & $108(12)$ \\
\hline
\end{tabular}

Composite digit-span scores and intelligence quotient (IQ) measures are from WAIS-III. See section on Behavioral Tests for full details. Mean scores are shown with standard deviations in brackets.

${ }^{\mathrm{a}}$ Maximum sequence length recalled over testing.

${ }^{\mathrm{b}}$ Differences between groups significant at $p<.05$. 
to orally recall the sequence with no delay period (immediate serial recall). The test is discontinued if both trials of a test item are recalled incorrectly (e.g., a failure to recall two 5-digit sequences correctly). Each correct trial is awarded a score of 1 , with a total maximum score of 16 points.

The digits-backward task has a similar format, consisting of seven levels, each containing two trials of the same length. The test starts with a two-digit sequence, which increases to a maximum length of eight digits. Participants are asked to recall digits in reverse order (for instance, if the tester said the sequence "2-4-1" then the correct response would be "1-4-2"). This test is also discontinued if both trials of a given item are recalled incorrectly. Each correct trial is awarded a score of 1 , with a maximum score of 14 points.

The scores for both tasks are added together to give an overall test score. The total maximum score is 30 points.

\section{Spoonerisms}

This test assesses the ability of participants to maintain two auditory words in STM, whereas the initial phonemes of each word pair are segmented and swapped to generate a verbal response. For example, the spoken stimulus basket-lemon is reproduced as lasket-bemon. This task, therefore, involves the segmentation and manipulation of phonemes and the phonological encoding of speech output responses before articulation. The materials for this test were taken from the phonological assessment battery (Frederickson, Frith, \& Reason, 1997). Two word pairs were used as practice examples at the start of the test; the remaining 10 word pairs were used for testing.

During this task, participants listened to an auditory recording of each word pair and repeated the stimuli heard after swapping the initial phonemes. Each correctly swapped word within a pair was given a score of 1 . Thus, the total maximum score on this test is 20 points. Successful performance on this task is dependent on both phonemic awareness and a participant's ability to retain representations of phonemically segmented words in STM. Thus, like the digit-span task, our supposition was that the spoonerisms task also loads on storage and maintenance components in STM. Therefore, we expected that the total score on this task would partially correlate with auditory STM capacity. Indeed, scores on the digit-span and spoonerisms tasks were significantly positively correlated for both dyslexics $[r(40)=0.318, p<.05]$ and typical adults $[r(34)=0.581, p<.001]$.

\section{Auditory Nonword Repetition}

Our stimuli were the same as those used in the nonword repetition task of Gathercole and Baddeley (1989). All participants listened to auditory recordings of 40 nonwords (e.g., glistering, doppelate), which they were asked to repeat back to the experimenter. The complexity of these items ranged from one to four syllables in length and according to whether they contained single consonants or consonant clusters. Stimuli consisted of eight sets of five items for each syllable length and consonant class. Items were presented in a fixed random order. Participants received a score of 1 point for each nonword pronounced correctly, with a total maximum score being 40 points. In comparison with the digit-span test, nonword repetition places higher demands on auditory speech perception and production planning but lower demands on auditory STM. By including nonword repetition scores in our analysis, we aimed to control for variance associated with auditory speech perception and production. Although the ability to repeat heard nonwords is affected by phonological storage capacity (Gathercole, 2006), we found the correlation between nonword repetition and digit-span to be weak in dyslexics $[r(40)=0.225, p=.162]$ and typical adults $[r(34)=0.036, p=.839]$. Moreover, the performance of our dyslexic group was not significantly different from the typical adults, with both groups achieving high scores on this task (see Table 1), although prior studies have used auditory nonword repetition to detect phonological processing deficits.

\section{Word Reading}

Reading scores were included to factor out reading ability within group. The test measures the ability of participants to convert orthography into phonology and requires speech production and phonological knowledge of visual word forms with minimal demands on auditory STM. We used the blue reading test from the Wide Range Achievement Test-3rd Edition (WRAT-3; Wilkinson, 1993). This test consists of 42 words and was computerized so that participants simply read each word as it was presented on-screen, pressing the spacebar on the keyboard to view the next word. Their responses were recorded, and the tester later marked whether the pronunciation of each word was correct or not. Correct responses were given a score of 1 , and incorrectly pronounced words or nonresponses were given a score of 0 . The maximum score was 42 points. By including reading scores in our analysis, we aimed to factor out reading ability within group and control for variance associated with phonological retrieval and speech production.

\section{Structural Imaging}

\section{Structural Image Acquisition}

Focal gray and white matter was estimated on the basis of T1-weighted anatomical whole brain images acquired using a Siemens Sonata 1.5-T MRI scanner (Siemans Medical Systems, Erlangen, Germany). A T1-weighted Modified Driven Equilibrium Fourier Transform sequence (Deichmann, Schwarzbauer, \& Turner, 2004) was used to acquire 176 sagittal partitions with an image matrix of $256 \times 224$, yielding a final resolution of $1 \mathrm{~mm}^{3}(\mathrm{TR} / \mathrm{TE} / \mathrm{TI}=12.24 \mathrm{msec} /$ 
$3.56 \mathrm{msec} / 530 \mathrm{msec}$ ). One T1 anatomical volume was acquired for each participant.

\section{Structural Image Analysis}

Scans were analyzed using SPM8 (Wellcome Department of Imaging Neuroscience, www.fil.ion.ucl.ac.uk/spm). Structural images were processed using the DARTEL toolbox available in SPM8 (Ashburner, 2007). DARTEL uses a more sophisticated registration model than previous approaches implemented in the SPM software (Ashburner, 2009). Structural images were first segmented in native space into gray and white matter. Gray and white matter images from a total of 172 scans in our database (including the 74 participants who took part in this study) were imported into DARTEL format. A template brain was then created in DARTEL using default parameter settings predefined within SPM8. This process iteratively matches selected images to a template generated by their own mean. There were six outer iterations, each consisting of three inner iterations. Over outer iterations, the parameter settings were as follows: $\mathrm{mu}=[4,2,1,0.05,0.25,0.25]$, lambda $=[2,1,0.5,0.25,0.125,0.125], \mathrm{id}=1 \mathrm{e}-006$, time steps $=[1,1,2,4,16,64]$, smoothing $=[16,8,4,2,1,0.5]$. The resulting flow fields containing deformation information generated by this process were then used to spatially normalize gray and white matter images to Montreal Neurological Institute (MNI) space. Following segmentation and normalization, the images can be either modulated or unmodulated. Modulated images are adjusted for the local gray or white matter volume identified in the normalization process by scaling the images by the Jacobian determinates of the deformation (i.e., by the degree of compression). The voxels in modulated images, therefore, provide an absolute measure of regional volume (Ashburner, 2009). In contrast, unmodulated images are not adjusted for volume, and therefore, each voxel provides an estimation of regional density (Mechelli, Price, Friston, \& Ashburner, 2005).

The results of VBM studies are expected to depend on whether modulated or unmodulated images are used. For example, if better auditory STM is associated with equivalent effects on (i) the probability of gray or white matter and (ii) the local volume (as measured by the degree of compression), then these effects could cancel out and be undetected when modulated images (probability/ compression) are used but they will be detected in the unmodulated images. On the other hand, if better auditory STM is associated with volume increases but not the probability of gray matter, then this will be detected in the modulated images but not the unmodulated images. Analyses based on modulated images are expected to be most sensitive to detecting volume changes associated with age or gender (e.g., Good et al., 2001). In language studies, however, we have previously found that our VBM analyses are more sensitive and more consistent across subject groups when we used unmodulated images than when we used modulated images (Richardson, Thomas,
Filippi, Harth, \& Price, 2010; Grogan, Green, Ali, Crinion, \& Price, 2009; Lee et al., 2007; Mechelli et al., 2004). This could be because the effects we are measuring are affecting both volume and the probability of gray matter. In all our analyses, we included age and gender as covariates to control for known differences in volume that might confound our results.

All normalized images were smoothed using an isotropic kernel of $8 \mathrm{~mm}$ at FWHM. Our main regression analysis (detailed below) was then run on both unmodulated and modulated gray and white matter images.

\section{Statistical Analyses of Structural Data}

Our main analysis used a factorial design in SPM8 in order carry out a regression analysis to identify the main effect of auditory STM capacity when each of the samples (dyslexic and typical adults) were modeled as separate groups within the same design matrix. This allowed us to estimate consistencies and inconsistencies across groups to provide a more accurate partitioning of variance related to auditory STM capacity. Scores from four behavioral tests were included as covariates in this analysis. The main effect of auditory STM capacity was modeled by the first two measures: (i) composite digit span (from the standard WAIS-III assessment) and (ii) spoonerisms. We used the composite digit-span measure on the basis of neuropsychological data indicating that combining forward and backward digitspan scores increases reliability of the digit-span measure (Wilde et al., 2004). Scores from tests of (iii) nonword repetition and (iv) word reading were included to factor out variance associated with speech perception, speech production, and reading skills within each group independently. Finally, age in years and gender were also included as covariates of no interest. The main effect of auditory STM capacity across group and for each group separately was tested using $t$ contrasts with an equal positive weighting on both composite digit-span and spoonerism measures.

\section{Confirmatory Analyses}

As composite digit-span and spoonerisms scores were used to identify an effect of auditory STM capacity in our main analysis, we carried out five additional regression analyses in SPM8 to assess the strength of the gray matter correlation for each covariate independently: (i) forward digit span, (ii) backward digit span, (iii) spoonerisms, (iv) nonword repetition, and (v) word reading. Each analysis included our full sample of 74 participants, in which dyslexic and typical groups were modeled separately. In addition, we report the effect of our main analysis (auditory STM above all other regressors) for the typical and dyslexic groups separately. Age in years and gender were included as covariates of no interest in all analyses. We report the $Z$ score for each covariate of interest for the peak within a 4-mm radius of the main effect of auditory STM identified in our main analyses. 


\section{Statistical Thresholds}

To identify the most salient effects of auditory STM capacity on regional gray and white matter, the statistical threshold for our regression analysis was set to $p<.05$ in height (the size of the peak effect), after family-wise correction for multiple comparisons across the whole brain. In our confirmatory analyses, we report the $Z$ and $T$ scores for each regressor separately in the voxels associated with auditory STM capacity in our main multiple regression analysis. As the confirmatory analyses are purely descriptive, we report significant and nonsignificant effects in the ROI, at $p<.05$ uncorrected.

\section{RESULTS}

In our analysis of gray matter density, using the unmodulated images, we identified a main effect of auditory STM capacity (as indexed by the main effect over digit-span and spoonerism performance), in a region in the left mid to posterior STS (henceforth referred to as left pSTS) at $[x=-56, y=-30, z=1]$ ( $Z$ score $=5.3,285$ voxels; $p<.05$, corrected). This result is shown in Figure 1 , which also illustrates its close proximity to that reported by Leff et al. (2009), in which gray matter density was positively correlated with forward digit-span scores in 210 stroke patients. No significant differences between groups were identified across the whole brain $(p>.05$ corrected) or in the left pSTS $(p>.05$ uncorrected). This is consistent with prior studies that have shown inconsistent structural brain differences between typical and dyslexic individuals that are only significant when small volumes of interest are used (Pernet, Andersson, Paulesu, \& Demonet, 2009; Richardson \& Price, 2009).

The effect of auditory STM on left pSTS gray matter density did not interact with group (dyslexic vs. typical adults) nor did group interact with the effect of digit-span on left pSTS gray matter density. However, an interaction between spoonerisms and group $[x=-57, y=-30, z=3$, $Z$ score $=4.4, p<.001$, uncorrected] indicated that the effect of spoonerisms was stronger in the typical adults than in the dyslexics. This might reflect the different range of scores in the two groups.

None of the above effects were significant when the analysis of gray matter volume was conducted using modulated images $(p>.05$, corrected). This is consistent with our prior studies that replicate the effects of language ability previously observed for gray matter density but not gray matter volume (Richardson et al., 2010; Grogan et al., 2009; Lee et al., 2007; Mechelli et al., 2004). Analyses of white matter images did not reveal any significant correlations with auditory STM capacity.

\section{Confirmatory Analyses}

The results of the confirmatory analyses can be seen in Table 2. This table shows the $T$ and $Z$ scores for each

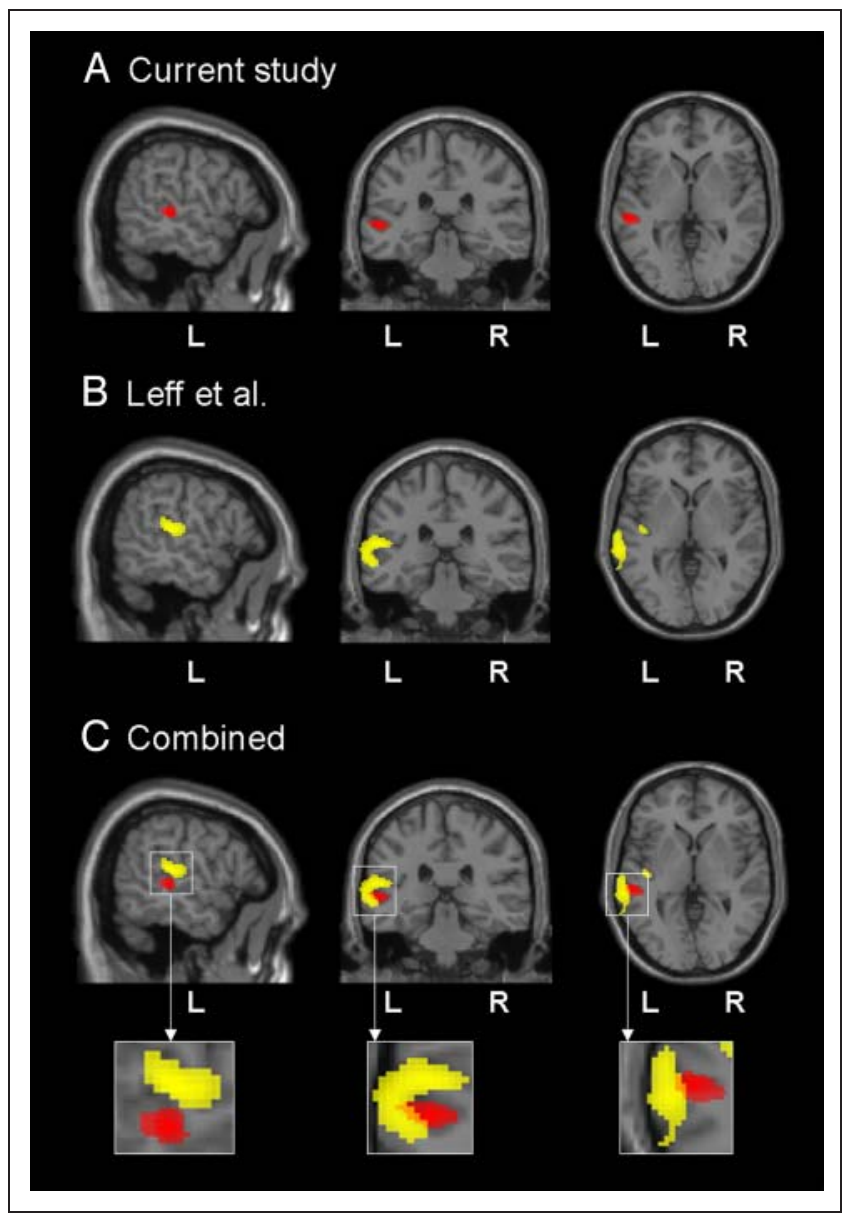

Figure 1. Location of area associated with auditory STM in the current study and that by Leff et al. (2009). (A) The effect of composite digit-span score (forward and backward) and spoonerisms on gray matter density in the left pSTS at the peak coordinates for this effect at $[x=-56, y=-30, z=1]$ (threshold $p=.001,250$ voxels). (B) The effect of digit-span identified in the patient study of Leff et al. (2009) at the same location (threshold $p=.0001,250$ voxels). (C) Results from the current study and the study of Leff et al. combined on the same images.

covariate in isolation in the left pSTS identified above. The $Z$ scores for forward digit span, backward digit span, and spoonerisms were all significant at $p<.05$, indicating that the effect identified in our main analysis was not driven by one particular measure (e.g., forward or backward digit span), but rather a composite effect of auditory STM capacity. This is illustrated in Figure 2, which shows the positive correlation between gray matter density at the peak voxel in the pSTS, for both digit-span measures and spoonerism scores. There was no correlation between nonword repetition or word reading scores and gray matter density in this region $(p>.05$, uncorrected). No additional regions were detected as significant at the whole-brain level in these analyses. Our separate analyses of groups indicated that an effect of auditory STM capacity was present in the same left pSTS region for both typical $(Z$ score $=2.5)$ and dyslexic $(Z$ score $=2.8)$ 
Table 2. Results from Confirmatory Analyses

\begin{tabular}{|c|c|c|c|}
\hline & $T$ & Z Score & $n$ Voxels \\
\hline Auditory STM capacity ${ }^{\mathrm{a}}$ & 6.0 & 5.3 & 285 \\
\hline Forward digit span & 2.4 & 2.4 & 26 \\
\hline Backward digit span & 3.2 & 3.1 & 51 \\
\hline Spoonerisms & 3.7 & 3.5 & 81 \\
\hline Nonword repetition & $n s$ & ns & - \\
\hline Word reading & ns & ns & - \\
\hline
\end{tabular}

Results show $T$ and $Z$ scores and $n$ voxels (at $p<.05$, peak within 4-mm radius of main effect) for the correlation between gray matter in left pSTS for each covariate included in the main analysis.

${ }^{\text {a }}$ Result is significant across the whole brain at $p<.05$, FWE corrected.

groups independently, but the result was much more significant when the two groups were combined to provide a wider range of scores.

\section{DISCUSSION}

The aim of this study was to identify the gray and white matter regions that provide the most robust prediction of auditory STM capacity in the healthy adult brain. We investigated this relationship using two groups of healthy adult participants: One group had a prior diagnosis of developmental dyslexia; the other included typical readers with no history of cognitive impairment. The inclusion of these two groups allowed us to test whether correlations between gray matter density and auditory STM were consistent across two different populations or whether the effect of auditory STM capacity differed in the dyslexics and typical adults. However, we found that auditory STM capacity was positively correlated with gray matter density in the left pSTS in both groups. This effect was significant at the whole-brain level in a multiple regression analysis in which variance associated with speech perception, speech production, and reading ability were also factored out. Confirmatory analyses demonstrated that this effect was present irrespective of whether auditory STM capacity was tested with forward digit span, backward digit span, or performance on the spoonerisms task-indicating that the effect we identified was not task specific.

Our result suggests that gray matter density in the left pSTS is a strong predictor of auditory STM capacity in the healthy adult brain. This effect may represent differences in cortical thickness or variance in sulcal morphology (Ashburner, 2009). This left pSTS region is also in proximity to the region identified by Leff and colleagues (2009), who found a positive correlation between digit span and structural integrity of the left pSTS/STG in stroke patients at $[x=-66, y=-32, z=4]$. This area extends to the lateral surface of STG, whereas the effect we observe in healthy subjects was within pSTS (see Figure 1). Together, these results suggest continuity in the relationship between auditory STM capacity and gray matter density in the left pSTS across healthy and patient populations. However, further studies are required to determine whether the lateral and medial parts of the pSTS/STG region have dissociable functions.

From a cognitive perspective, it is interesting to consider what role the left pSTS might play in auditory STM. We can address this issue by examining the functional properties of this region during language and verbal working memory tasks. Activation in the same part of the left pSTS has been
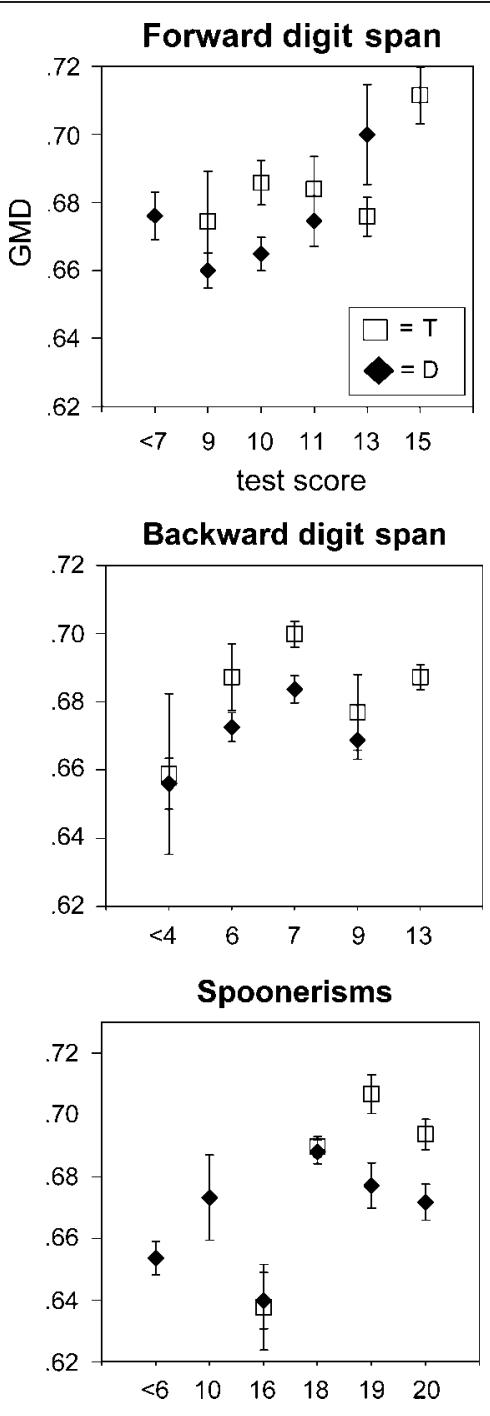

Figure 2. The relationship between gray matter density (GMD) and individual test scores. The correlation between gray matter density probability at the peak voxel in the left pSTS at $[x=-56, y=-30$, $z=1]$ and tests indexing auditory STM capacity. Results are displayed according to participant group ( $\mathrm{T}=$ typical; $\mathrm{D}=$ dyslexic). Data points represent mean of gray matter density values across participants according to test score. 
associated with a range of acoustic stimuli, from music and environmental sounds to sentences, words, pseudowords, and vocoded speech (Price, Thierry, \& Griffiths, 2005; Hickok et al., 2003; Thierry, Giraud, \& Price, 2003; Wise et al., 2001; Binder et al., 2000; Scott, Blank, Rosen, \& Wise, 2000). More specifically, Binder and colleagues (2000) have reported a region in the left pSTS activated for words $[x=$ $-60, y=-34, z=1]$, pseudowords $[x=-58, y=-34$, $z=3]$, and reversed speech $[x=-62, y=-34, z=3]$ relative to tones (coordinates converted to MNI space). These coordinates are in a similar location to the peak coordinates we report for the correlation between auditory STM capacity and gray matter density at $[x=-56$, $y=-30, z=1$ ] and where Leff et al. (2009) report the same correlation in brain-damaged patients at $[x=-66$, $y=-32, z=4]$.

Recent studies also suggest that the left pSTS responds more strongly when acoustic stimuli can be categorized as meaningful in some way. For instance, this region shows increased activation at $[x=-54, y=-37, z=$ -1 ] when participants are trained to categorize complex artificial nonspeech sounds (Leech, Holt, Devlin, \& Dick, 2009) and phonetic sine-wave sounds at $[x=-59, y=$ $-34, z=4$ ] (coordinates converted to MNI space; Desai, Liebenthal, Waldron, \& Binder, 2008). The left pSTS is also activated during tasks that involve not only the perception but also the retrieval of words from memory. For instance, Wise and colleagues (2001) reported common activation at $[x=-63, y=-34, z=2]$, for both hearing and retrieving words. A similar region has also been reported in a study by Hickok and colleagues (2003), which showed activation during auditory perception and retention of nonsense sentences at $[x=-60$, $y=-31, z=2]$ and melodic tonal sequences at $[x=$ $-60, y=-33, z=3$ ] (coordinates converted to MNI space). A study of verbal working memory by Rypma and colleagues (1999) has also identified activation in the left pSTS during high-loading verbal working memory conditions at $[x=-54, y=-31, z=-1]$ (coordinates converted to MNI space).

The functional imaging literature, therefore, suggests that the left pSTS is involved in the initial formation of internal representations of perceived stimuli for the purposes of recognition as well as being involved in perceptual processing. However, the left pSTS region we are referring to is distinct from the more dorsal and posterior STG region associated with speech production (Graves, Grabowski, Mehta, \& Gupta, 2008; Graves, Grabowski, Mehta, \& Gordon, 2007; Wise et al., 2001), which is also referred to in the literature as area Spt (Hickok et al., 2003). Buchsbaum, Olsen, Koch, and Berman (2005) suggest that both pSTS and Spt are engaged by STM but have different roles. They linked area Spt with sustained rehearsal, and the left STS/STG with memory retrieval. They suggest that the STS/STG region may be associated with echoic memory - a form of STM that retains auditory sound sequence information for durations of up to several seconds (Cowan, 1984). Consistent with this perspective, a later study by Strand, Forssberg, Klingberg, and Norrelgen (2008), which used pseudowords as stimuli, linked the left pSTS at $[x=-48, y=-39, z=3]$ with the "maintenance" phase of their auditory STM task. Wilson, Isenberg, and Hickok (2009) also associate the left pSTS at $[x=$ $-56, y=-32, z=-4]$ with the storage of phonological information.

Structural imaging studies have also found a link between the pSTS and verbal ability in children (Eckert et al., 2008) and vocabulary knowledge across lifespan at $[x=-48, y=-36, z=6]$ (Richardson et al., 2010). Prior evidence, therefore, supports the view that there is an overlap between the mechanisms that support language processing and STM (Acheson \& MacDonald, 2009b; Leff et al., 2009; D'Esposito, 2007). Some structural imaging studies of developmental dyslexia have identified reduced gray matter in posterior temporal regions (Steinbrink et al., 2008; Hoeft et al., 2007; Silani et al., 2005; Brambati et al., 2004). However, these structural differences are inconsistent across studies and only identified when small volumes of interest are used (Pernet et al., 2009; Richardson \& Price, 2009). In the context of the present study, we did not identify any differences in gray matter density across groups or in the pSTS region we have associated with STM capacity.

In addition to the temporal regions described above, functional imaging studies of verbal STM report activation across a range of parietal sites as well as inferior and middle frontal regions (Strand et al., 2008; Buchsbaum et al., 2005; Crottaz-Herbette et al., 2004; Gruber \& von Cramon, 2003; Henson et al., 2000; Jonides et al., 1998; Awh et al., 1996; Salmon et al., 1996; Smith et al., 1995, 1996; Paulesu et al., 1993). These findings have been supported by a patient study showing a positive correlation between performance on the digits-backward task and reduced gray matter volume in a large area of the left inferior and middle frontal gyri and a small left inferior parietal region (Amici et al., 2007). Gruber and von Cramon (2003) suggest that this prefrontalparietal network supports phonological storage under conditions where speech-based rehearsal is insufficient to solve the memory task. This would explain why we did not find a correlation between frontal and parietal gray matter and digit-span performance in healthy participants.

To conclude, we show that gray matter density in pSTS predicts auditory STM capacity in the healthy brain of developmental dyslexics and typical adults. This provides an interesting continuum with the prior demonstration that gray matter density in the same region correlates with auditory STM capacity in the damaged brain (Leff et al., 2009). Future investigation is now required to understand how the pSTS region interacts with other working memory areas during linguistic and nonlinguistic tasks and whether the pSTS we refer to can be partitioned into functionally specialized subdivisions. 


\section{APPENDIX}

Correlations between Behavioral Test Scores Used in Regression Analyses

\begin{tabular}{|c|c|c|c|}
\hline \multirow{2}{*}{$\begin{array}{l}\text { Pairs of Regressors Tested } \\
\text { Using Simple Correlation }\end{array}$} & \multicolumn{3}{|c|}{ Group } \\
\hline & All & Typical & Dyslexic \\
\hline Digit span and spoonerisms & $.466 * *$ & $.581 * *$ & $.318^{*}$ \\
\hline $\begin{array}{l}\text { Digit span and nonword } \\
\text { repetition }\end{array}$ & .188 & .037 & .224 \\
\hline Digit span and word reading & $.552 * *$ & $.558 * *$ & $.363^{*}$ \\
\hline $\begin{array}{l}\text { Spoonerisms and nonword } \\
\text { repetition }\end{array}$ & $.337^{* *}$ & .207 & $.346 *$ \\
\hline Spoonerisms and word reading & $.417^{* *}$ & $.628 * *$ & .268 \\
\hline $\begin{array}{l}\text { Nonword repetition and word } \\
\text { reading }\end{array}$ & .200 & .117 & .182 \\
\hline
\end{tabular}

\section{Acknowledgments}

This research was funded by the Wellcome Trust. We thank Sarah White for her help preparing some of the stimuli.

Reprint requests should be sent to Dr. Fiona Richardson, Wellcome Trust Centre for Neuroimaging, UCL, 12 Queen Square, London, WC1N 3BG, UK, or via e-mail: fiona.richardson@ucl.ac.uk.

\section{REFERENCES}

Acheson, D. J., Hamidi, M., Binder, J. R., \& Postle, B. R. (2011). A common neural substrate for language production and verbal working memory. Journal of Cognitive Neuroscience, 23, 1358-1367.

Acheson, D. J., \& MacDonald, M. C. (2009a). Twisting tongues and memories: Explorations of the relationship between language production and verbal working memory. Journal of Memory and Language, 60, 329-350.

Acheson, D. J., \& MacDonald, M. C. (2009b). Verbal working memory and language production: Common approaches to the serial ordering of verbal information. Psychological Bulletin, 135, 50-68.

Amici, S., Brambati, S. M., Wilkins, D. P., Ogar, J., Dronkers, N. L., Miller, B. L., et al. (2007). Anatomical correlates of sentence comprehension and verbal working memory in neurodegenerative disease. Journal of Neuroscience, 27, 6282-6290.

Ashburner, J. (2007). A fast diffeomorphic image registration algorithm. Neuroimage, 38, 95-113.

Ashburner, J. (2009). Computational anatomy with the SPM software. Magnetic Resonance Imaging, 27, $1163-1174$.

Awh, E., Jonides, J., Smith, E. E., Schumacher, E. H., Koeppe, R. A., \& Katz, S. (1996). Dissociation of storage and rehearsal in verbal working memory: Evidence from positron emission tomography. Psychological Science, 7, 25-31.

Baddeley, A. (2003). Working memory: Looking back and looking forward. Nature Reviews Neuroscience, 4 , 829-839.
Baddeley, A. D., \& Hitch, G. J. (1974). Working memory. In G. Bower (Ed.), The psychology of learning and motivation (Vol. 7, pp. 47-90). New York: Academic Press.

Binder, J. R., Frost, J. A., Hammeke, T. A., Bellgowan, P. S. F., Springer, J. A., Kaufman, J. N., et al. (2000). Human temporal lobe activation by speech and nonspeech sounds. Cerebral Cortex, 10, 512-528.

Brambati, S. M., Termin, C., Ruffino, M., Stella, G., Fazio, F., Cappa, S. F., et al. (2004). Regional reductions of gray matter volume in familial dyslexia. Neurology, 63, 742-745.

Buchsbaum, B. R., \& D'Esposito, M. (2008). The search for the phonological store: From loop to convolution. Journal of Cognitive Neuroscience, 20, 762-778.

Buchsbaum, B. R., Olsen, R. K., Koch, P., \& Berman, K. F. (2005). Human dorsal and ventral auditory streams subserve rehearsal-based echoic processes during verbal working memory. Neuron, 48, 687-697.

Buchsbaum, B. R., Padmanabhan, A., \& Berman, K. F. (2011). The neural substrates of recognition memory for verbal information: Spanning the divide between short- and long-term memory. Journal of Cognitive Neuroscience, 23, 978-991.

Bunge, S. A., Ochsner, K. N., Desmond, J. E., Glover, G. H., \& Gabrieli, J. D. E. (2001). Prefrontal regions involved in keeping information in and out of mind. Brain, 124, 2074-2086.

Chen, S. H. A., \& Desmond, J. E. (2005). Cerebrocerebellar networks during articulatory rehearsal and verbal working memory tasks. Neuroimage, 24, 332-338.

Cohen, J. D., Perlstein, W. M., Braver, T. S., Nystrom, L. E., Noll, D. C., Jonides, J., et al. (1997). Temporal activation of brain dynamics during a working memory task. Nature, 386, 604-608.

Cowan, N. (1984). On short and long auditory stores. Psychological Bulletin, 96, 341-370.

Crottaz-Herbette, S., Anagnoson, R. T., \& Menon, V. (2004) Modality effects in verbal working memory: Differential prefrontal and parietal responses to auditory and visual stimuli. Neuroimage, 21, 340-351.

Deichmann, R., Schwarzbauer, C., \& Turner, R. (2004) Optimisation of the 3D MDEFT sequence for anatomical brain imaging: Technical implications at $1.5 \mathrm{~T}$ and $3 \mathrm{~T}$. Neuroimage, 21, 757-767.

Desai, R., Liebenthal, E., Waldron, E., \& Binder, J. E. (2008). Left posterior temporal regions are sensitive to auditory categorization. Journal of Cognitive Neuroscience, 20, 1174-1188.

D'Esposito, M. (2007). From cognitive to neural models of working memory. Philosophical Transactions of the Royal Society of London, Series B, Biological Sciences, 362, 761-772.

Eckert, M. A., Lombardino, L. J., Walczak, A. R., Bonihla, L., Leonard, C. M., \& Binder, J. R. (2008). Manual and automated measures of superior temporal gyrus asymmetry: Concordant structural predictors of verbal ability in children. Neuroimage, 41, 813-822.

Ericsson, A., Chase, W. G., \& Faloon, S. (1980). Acquisition of a memory skill. Science, 208, 1181-1182.

Frederickson, N., Frith, U., \& Reason, R. (1997). Phonological assessment battery: Standardisation edition. Windsor: NFER-Nelson.

Gathercole, S. E. (2006). Nonword repetition and word learning: The nature of the relationship. Applied Psycholinguistics, 27, 513-543.

Gathercole, S. E., \& Baddeley, A. D. (1989). Evaluation of the role of phonological STM in the development of vocabulary in children: A longitudinal study. Journal of Memory and Language, 28, 200-213. 
Gathercole, S. E., Alloway, T. P., Willis, C., \& Adams, A. M. (2006). Working memory in children with reading disabilities Journal of Experimental Child Psychology, 93, 265-281.

Good, C. D., Johnsrude, I. S., Ashburner, J., Henson, R. N. A., Friston, K., \& Frackowiak, R. S. J. (2001). A voxel-based morphometric study of ageing in 465 normal adult human brains. Neuroimage, 14, 21-36.

Graves, W. W., Grabowski, T. J., Mehta, S., \& Gordon, J. K. (2007). A neural signature of phonological access: Distinguishing the effects of word frequency from familiarity and length in overt picture naming. Journal of Cognitive Neuroscience, 19, 617-631.

Graves, W. W., Grabowski, T. J., Mehta, S., \& Gupta, P. (2008). The posterior superior temporal gyrus participates in accessing lexical phonology. Journal of Cognitive Neuroscience, 20, 1698-1710.

Grogan, A., Green, D. W., Ali, N., Crinion, J. T., \& Price, C. J. (2009). Structural correlates of semantic and phonemic fluency ability in first and second languages. Cerebral Cortex, 19, 2690-2698.

Gruber, O., \& von Cramon, D. Y. (2003). The functional neuroanatomy of human working memory revisited. Evidence from 3-T fMRI studies using classical domain interference tasks. Neuroimage, 19, 797-809.

Hatcher, J., Snowling, M. J., \& Griffiths, Y. (2002). Cognitive assessment of dyslexic students in higher education. British Journal of Educational Psychology, 72, 119-133.

Henson, R. N., Burgess, N., \& Frith, C. D. (2000). Recoding, storage, rehearsal and grouping in verbal short-term memory: An fMRI study. Neuropsychologia, 38, 426-440.

Hickok, G., Buchsbaum, B., Humphries, C., \& Muftuler, T. (2003). Auditory-motor interaction revealed by fMRI: Speech, music and working memory in area Spt. Journal of Cognitive Neuroscience, 15, 673-682.

Hoeft, F., Meyler, A., Hernandez, A., Juel, C., Taylor-Hill, H., Martindale, J. L., et al. (2007). Functional and morphometric brain dissociation between dyslexia and reading ability. Proceedings of the National Academy of Sciences, U.S.A., 4, 4234-4239.

Jonides, J., Schumacher, E. H., Smith, E. E., Koeppe, R. A., Awh, E., Reuter-Lorenz, P. A., et al. (1998). The role of the parietal cortex in verbal working memory. Journal of Neuroscience, 18, 5026-5034.

Kimberg, D. Y., Coslett, H. B., \& Schwartz, M. F. (2007). Power in voxel-based lesion-symptom mapping. Journal of Cognitive Neuroscience, 19, 1067-1080.

Kirschen, M. P., Chen, S. H. A., Schraedley-Desmond, P., \& Desmond, J. E. (2005). Load- and practice-dependent increases in cerebro-cerebellar activation in verbal working memory: An fMRI study. Neuroimage, 24, 462-472.

Koelsch, S., Schulze, K., Sammler, D., Fritz, T., Müller, K., \& Gruber, O. (2009). Functional architecture of verbal and tonal working memory: An fMRI study. Human Brain Mapping, 30, 859-873.

Lee, H. L., Devlin, J. T., Shakeshaft, C., Stewart, L. H., Brennan, A., Glensman, J., et al. (2007). Anatomical traces of vocabulary acquisition in the adolescent brain. Journal of Neuroscience, 27, 1184-1189.

Leech, R., Holt, L. L., Devlin, J. T., \& Dick, F. (2009).

Expertise with artificial nonspeech sounds recruits speech-sensitive cortical regions. Journal of Neuroscience, 29, 5234-5239.

Leff, A., Schofield, T., Crinion, J., Seghier, M., Grogan, A., Green, D., et al. (2009). The left superior temporal gyrus is a shared substrate for auditory short-term memory and speech comprehension: Evidence from 210 stroke patients. Brain, 132, 3401-3410.
Mechelli, A., Crinion, J. T., Noppeney, U., O’Doherty, J., Ashburner, J., Frackowiak, R. S. J., et al. (2004).

Neurolinguistics: Structural plasticity in the bilingual brain. Nature, 431, 757.

Mechelli, A., Price, C. J., Friston, K., \& Ashburner, J. (2005). Voxel-based morphometry of the human brain: Methods and applications. Current Medical Imaging Reviews, 1, 105-113

Miller, G. A. (1956). The magical number seven plus or minus two: Some limits on our capacity for processing information. Psychological Review, 63, 81-97.

Page, M. P. A., Madge, A., Cumming, N., \& Norris, D. G. (2007). Speech errors and the phonological similarity effect in short-term memory: Evidence suggesting a common locus. Journal of Memory and Language, 56, 49-64.

Paulesu, E., Frith, C. D., \& Frackowiak, R. S. (1993). The neural correlates of the verbal component of working memory. Nature, 362, 342-345.

Pernet, C., Andersson, J., Paulesu, E., \& Demonet, J. F. (2009). When all hypotheses are right: A multifocal account of dyslexia. Human Brain Mapping, 30, 2278-2292.

Price, C. J., Thierry, G., \& Griffiths, T. (2005). Speech-specific auditory processing: Where is it? Trends in Cognitive Sciences, 9, 271-276.

Ravizza, S. M., Delgado, M. R., Chein, J. M., Becker, J. T., \& Fiez, J. A. (2004). Functional dissociations within the inferior parietal cortex in verbal working memory. Neuroimage, 22, 562-573.

Richardson, F. M., \& Price, C. J. (2009). Structural MRI studies of language function in the undamaged brain. Brain Structure and Function, 213, 511-523.

Richardson, F. M., Thomas, M. S. C., Filippi, R., Harth, H., \& Price, C. J. (2010). Contrasting effects of vocabulary knowledge on temporal and parietal brain structure across lifespan. Journal of Cognitive Neuroscience, 22, 943-954.

Rudrauf, D., Mehta, S., Bruss, J., Tranel, D., Damasio, H., \& Grabowski, T. J. (2008). Thresholding lesion overlap difference maps: Application to category-related naming and recognition deficits. Neuroimage, 41, 970-984.

Rypma, B., Prabhakaran, V., Desmond, J. E., Glover, G. H., \& Gabrieli, J. D. E. (1999). Load-dependent roles of frontal brain regions in the maintenance of working memory. Neuroimage, 9, 216-226.

Salmon, E., Van der Linden, M., Collette, F., Delfiore, G., Maquet, P., Degueldre, C., et al. (1996). Regional brain activity during working memory tasks. Brain, 119, 1617-1625.

Scott, S. K., Blank, S. C., Rosen, S., \& Wise, R. J. S. (2000) Identification of a pathway for intelligible speech in the left temporal lobe. Brain, 123, 2400-2406.

Silani, G., Frith, U., Demonet, J.-F., Fazio, F., Perani, D., Price, C., et al. (2005). Brain abnormalities underlying altered activation in dyslexia: A voxel based morphometry study. Brain, 128, 2453-2461.

Smith, E. E., Jonides, J., \& Koeppe, R. A. (1996). Dissociating verbal and spatial working memory using PET. Cerebral Cortex, 6, 11-20.

Smith, E. E., Jonides, J., Koeppe, R. A., Awh, E., Schumacher, E., \& Minoshima, S. (1995). Spatial vs. object working memory: PET investigations. Journal of Cognitive Neuroscience, 7, 337-358.

Snowling, M. J., Nation, K., Moxham, P., Gallagher, A., \& Frith, U. (1997). Phonological processing deficits in dyslexic students: A preliminary account. Journal of Research in Reading, 20, 31-34.

Steinbrink, C., Vogt, K., Kastrup, A., Müller, H.-P., Juengling, F. D., Kassubek, J., et al. (2008). The contribution of white 
and gray matter differences to developmental dyslexia: Insights from DTI and VBM at 3.0T. Neuropsychologia, 46, 3170-3178.

Strand, T., Forssberg, H., Klingberg, T., \& Norrelgen, F. (2008). Phonological working memory with auditory presentation of pseudowords-An event related fMRI study. Brain Research, 1212, 48-54.

Thierry, G., Giraud, A.-L., \& Price, C. J. (2003). Hemispheric dissociation in access to the human semantic system. Neuron, 38, 499-506.

Thierry, G., Ibarrola, D., Démonet, J.-F., \& Cardebat, D. (2003). Demand in verbal working memory delays hemodynamic response in inferior prefrontal cortex. Human Brain Mapping, 19, 37-46.
Wechsler, D. (1998). Wechsler Adult Intelligence Scale-3rd Edition. London: Harcourt Assessment.

Wilde, N. J., Strauss, E., \& Tulsky, D. S. (2004). Memory span on the Weschler scales. Journal of Clinical and Experimental Neuropsychology, 26, 539-549.

Wilkinson, G. S. (1993). Wide Range Achievement Test-Revision 3. Wilmington, DE: Jastak Association.

Wilson, S. M., Isenberg, A. L., \& Hickok, G. (2009). Neural correlates of word production stages by parametric modulation of psycholinguistic variables. Human Brain Mapping, 30, 3596-3608.

Wise, R. J. S., Scott, S. K., Blank, S. C., Mummary, C. J., Murphy, K., \& Warburton, E. A. (2001). Separate neural subsystems within "Wernicke's area." Brain, 124, 83-95. 